\title{
Nosocomial Transmission OF TRICHOPHYTON TONSURANS TINEA CORPORIS IN A REHABILITATION HOSPITAL
}

\author{
Steven M. Lewis, MD, FACP; Barbara G. Lewis, MS
}

\begin{abstract}
OBJECTIVE: Investigate the nosocomial transmission of Trichophyton tonsurans tinea corporis.

DESIGN: Descriptive study of a nosocomial epidemic of tinea corporis. facility.

SETTING: A free-standing inpatient rehabilitation

PARTICIPANTS: Patients and healthcare workers present on an inpatient rehabilitation ward at the time of transmission of tinea corporis.

RESULTS: T tonsurans tinea corporis was transmitted

from one patient to four healthcare workers despite early diagnosis and treatment. Infection rates for healthcare workers having major, moderate, and minor contact with the index case were $30 \%, 17 \%$, and $0 \%$, respectively (overall rate, $25 \%$ ).

CONCLUSIONS: This study identifies rehabilitation inpatients as another population in which nosocomial transmission of $T$ tonsurans tinea corporis can occur. The high attack rate and transmission, despite early diagnosis and treatment, emphasizes the need for isolation precautions (Infect Control Hosp Epidemiol 1997;18:322-325).
\end{abstract}

Dermatophytosis due to Trichophyton tonsurans is the most common cause of tinea in children, 1,2 has an asymptomatic carrier state, 3,4 is associated with epidemics, ${ }^{5-11}$ spreads to adults, ${ }^{4,9-11}$ and can have a high attack rate among exposed adults. 4,11 Despite these features, there have been few reports of nosocomial transmission of $T$ tonsurans. ${ }^{10,11}$ We report an outbreak of $T$ tonsurans tinea corporis in a rehabilitation hospital.

\section{METHODS}

\section{Description of Outbreak}

Index case. A 6-year-old white male was admitted to the pediatric unit of a 110-bed, free-standing rehabilitation hospital for comprehensive inpatient rehabilitation after 11 weeks of hospitalization for anoxic encephalopathy and $20 \%$ body surface area variable-thickness burns incurred in a house fire. The burns involved the face, anterior torso, and anterior thighs. The buttocks were not involved. After 5 weeks of rehabilitation, he began going home on weekly therapeutic passes during which time he had exposure to other children, none of whom were known to have any skin lesions. During week 9 of rehabilitation, a $5-\mathrm{cm}$, red, slightly vesicular, weeping lesion was noted on his left buttock during a diaper change. It was diagnosed as tinea corporis by his pediatrician. No confirmatory diagnostic tests were performed. Topical clotrimazole was started. In accordance with the Centers for Disease Control and Prevention (CDC) guidelines, no isolation was instituted. The lesion was contained by his diaper, which he wore at all times. He was noted by nurses to scratch the lesion intermittently.

Three days after the initial lesion appeared, it showed central clearing with peripheral expansion, and a second lesion $2 \mathrm{~cm}$ in diameter appeared lateral to the first lesion. Six days later, three new lesions, each approximately $1 \mathrm{~cm}$ in diameter, appeared on the right buttock. All lesions were treated with clotrimazole, and, by 3 weeks, all lesions were almost completely resolved. The patient was discharged on clotrimazole after 12 weeks of rehabilitation and subsequently experi-

From the Infection Control Department, Marianjoy Rehabilitation Hospital and Clinics, Wheaton, Illinois.

Presented at the Annual Meeting of the Association of Practitioners of Infection Control, Las Vegas, Nevada, May 1986.

Address reprint requests to Steven M. Lewis, MD, 1725 South St, Geneva, IL 60134.

95-OA-037. Lewis SM, Lewis BG. Nosocomial transmission of Trichophyton tonsurans tinea corporis in a rehabilitation hospital. Infect Control Hosp Epidemiol 1997;18:322-325. 
enced complete resolution of all lesions, with no recurrences after 5 months of follow-up.

Secondary cases. Approximately 3 weeks after the patient was discharged, or 6 to 7 weeks after the first tinea corporis lesion was noted, three healthcare workers developed red, circular, slightly vesicular lesions on the radial aspect of the distal left upper extremity, all occurring within an area that extended no more than 6 in up from the most distal lesion on the dorsum of the hand at the base of the thumb. A fourth healthcare worker developed a similar lesion on the left side of the chin. All four were diagnosed as having tinea corporis by an infectious disease physician.

\section{Evaluation of Outbreak}

Evaluation of infected healthcare workers. Each healthcare worker diagnosed with tinea corporis was examined and interviewed, and data were collected regarding prior history of skin lesions, exposure to other sources of tinea, and the frequency, duration, and circumstances of contact with the index case. For three healthcare workers, lesions were scraped with a scalpel, and the scrapings were treated with $10 \%$ potassium hydroxide and examined microscopically. For two healthcare workers, lesions were scraped and the scrapings used to inoculate Sabouraud's dextrose agar plates with cycloheximide-chloramphenicol and incubated at $25^{\circ} \mathrm{C}$. Growth on the plates was identified as Trichophyton tonsurans microscopically from tease mounts and microslide culture mounts prepared with lactophenol aniline (cotton) blue. Identification was verified by the mycology laboratory of the Illinois Department of Public Health.

Determination and stratification of infection rate for exposed population. All healthcare workers who provided patient care for the index case were evaluated for skin lesions. We identified all patient-care activities involving direct physical contact with the index case. These included bathing, dressing, diaper changes, transfers, and multiple therapy modalities. We then evaluated healthcare workers with regard to the extent and frequency with which they participated in these activities. Healthcare workers then were stratified into three categories of contact magnitude designated as "major," "moderate," and "minimal." Major-contact healthcare workers engaged frequently in most or all of the contact activities multiple times per day. Moderate-contact healthcare workers engaged in many of the contact activities, but only two or three times per week. The minimal-contact group had little or no direct physical contact with the index case.

All patients on the same patient-care unit as the index case were evaluated for skin lesions.
Determination of mode of transmission. The location of the lesions on the infected healthcare workers was noted and the care of the index patient evaluated to identify how the involved skin of the healthcare workers came in contact with the index patient.

\section{Estimating the Incubation Period}

The incubation period was defined as the time between the 9-day interval when lesions were appearing on the index patient and the time when healthcare workers developed lesions.

\section{RESULTS}

Four healthcare workers were identified with lesions typical of tinea corporis. Scrapings from three lesions were examined microscopically and all were positive. T tonsurans was grown in culture from lesion scrapings from two employees. No specimens for confirmatory testing yielded a negative result. No additional lesions or infected healthcare workers were detected with 3 months of follow-up.

None of the infected healthcare workers had any prior history of tinea or any skin condition that might have a similar appearance. None of the four infected healthcare workers had any history of exposure to tinea other than to the index case. All identified lesions were treated with topical clotrimazole, and all resolved promptly, with no additional lesions or relapses during 3 months of follow-up.

We identified 30 healthcare workers involved in delivering care to the index case, including 23 nurses, one physical therapist, two occupational therapists, one recreational therapist, one speech therapist, one psychologist, and one physician. Evaluation of the activities of these employees identified 10 with major contact, 7 with moderate contact, and 14 with minimal contact. In the major contact group, three healthcare workers developed infection, for an infection rate of $33 \%$. In the moderate contact group, there was one infection, for an infection rate of $17 \%$. There were no infections in the minimal contact group. The four infected healthcare workers consisted of two nurses, one occupational therapist, and one speech therapist. Among all healthcare workers with identifiable physical contact with the index case, the infection incidence was $25 \%$. There were no patients identified as having tinea other than the index case.

All healthcare worker lesions were located at sites that had been in contact with the index patient. The index patient transfers between bed and wheelchair were done by facing the patient, reaching around the torso, placing a hand on the sacrum or buttocks, then lifting and pivoting. The patient's room configuration was such that these transfers could be 
accomplished in only one position, with the left hand reaching under the patient. This procedure would bring the distal left arm, wrist, and hand into contact with the area where the patient's lesions were located. Two of the three infected healthcare workers that had left upper extremity lesions performed patient transfers several times per day. Transfers also brought the left side of the chin into contact with the patient's scalp, and the healthcare worker with a lesion on the left side of the chin performed transfers. The other healthcare worker with a left upper extremity lesion did not do patient transfers, but had frequent daily contact with the index case, including bathing, diaper changing, linen changing, and positioning in bed, and was left handed.

The incubation period was estimated to be 5 to 7 weeks.

\section{DISCUSSION}

This is the third report of transmission of $T$ tonsurans in a hospital setting and the first report involving a rehabilitation hospital. There have been six prior reports of nosocomial tinea corporis transmission, of which two were due to $T$ tonsurans, ${ }^{10,11}$ two due to Trichophyton rubrum, 12,13 and two due to Microsporum canis. 14,15

The outbreak that we report is similar to prior reports in several respects. The index case was a patient $10-15$ who was either geriatric $10,12-14$ or pediatric 12,13 and whose care involved considerable direct contact. The index case lesions involved the buttocks in an incontinent patient. ${ }^{12,13}$ The transmission to healthcare workers appeared to be primarily by direct contact with the index case ${ }^{10,11,14}$ and affected the exposed glabrous skin, especially of the arms. $11,14,15$ The attack rate was high among individuals with a recognizable risk for contact, 11,13 and the risk could be stratified based on extent of direct contact. 11

$T$ tonsurans has been reported previously to cause epidemics in multiple settings, including family units, ${ }^{5}$ residential schools, ${ }^{6}$ daycare centers, ${ }^{7}$ high school wrestlers, ${ }^{8}$ the general public, ${ }^{9}$ a nursing home for the elderly, ${ }^{10}$ and a hospital pediatric ward. ${ }^{11}$ Direct contact with the index case has been the principle risk factor for transmission to healthcare workers, and extensive physical contact is intrinsic to the care of rehabilitation inpatients.

Prior reports have described transmission to other patients. ${ }^{10,12,14}$ We detected no transmission to patients. Our index patient had a private room, shared staff with other patients, and used common areas for therapies, but did not have direct physical contact with other patients. This amount of physical isolation, combined with early recognition and treatment, was sufficient to prevent transmission to other patients.

In previous reports of nosocomial tinea outbreaks, transmission to healthcare workers has been related principally to direct contact, with lesions appearing on the exposed glabrous skin, especially of the hands and arms. ${ }^{11,14,15}$ The attempt in this report to relate lesion location to specific patient-care activities is conjectural, but emphasizes that all healthcare worker lesions involved unprotected skin that had been in direct physical contact with the index case.

Previous reports have not been able to determine the incubation period for nosocomially transmitted $T$ tonsurans. Because the index case had prompt recognition, treatment, and resolution of clinical tinea, we can estimate that the minimal incubation period was 5 to 7 weeks. It is possible that transmission to healthcare workers occurred before the onset of clinical illness in the index case and that the incubation period is longer.

The most important aspect of this report is that we observed a high infection rate despite early recognition and treatment of the index case. In prior reports of nosocomial transmission, a delay in diagnosis was a factor contributing to transmission. 11,13,15 Our index case developed new lesions over a 9-day interval, indicating that normal-appearing skin was infected when the initial lesions were recognized. The index patient's lesions resolved with treatment, but did so gradually over a 3 -week period, during which time they still may have been infectious. Concern that tinea corporis may be transmitted by either clinically uninvolved skin or partially treated lesions should be considered when taking precautions to prevent transmission. Avoiding contact with obvious lesions may not be a sufficient precaution, and the duration of treatment needed to prevent transmission is not known. Prior CDC Isolation Precautions Guidelines ${ }^{16}$ do not advise any type of precautions for tinea infections. Under the recently adopted CDC "Guideline for Isolation Precautions in Hospitals,"17 tinea infections are handled with Standard Precautions, which include precautions for direct contact with nonintact skin and for handling linens and patient-care equipment. The experience reported here indicates that, when caring for patients with $T$ tonsurans tinea corporis, all exposed skin that could come in contact with the index case should be covered, even for contact with skin that appears clinically normal. 


\section{REFERENCES}

1. Prevost E. Nonflourescent tinea capitis in Charleston, SC. JAMA 1979;242:1765-1767.

2. Ravits MS, Himmelstein R. Tinea capitis in the New York City area. Arch Dermatol 1983;119:532-533.

3. Sharma V, Hall JC, Knapp JF, Sumandeep S, Galloway D, Babel DE. Scalp colonization by Trichophyton tonsurans in an urban pediatric clinic. Arch Dermatol 1988;124:1511-1513.

4. Babel DE, Baughman SA. Evaluation of the adult carrier state in juvenile tinea capitis caused by Trichophyton tonsurans. J Am Acad Dermatol 1989;21:1209-1212.

5. Kligman AM, Constant ER. Family epidemic of tinea capitis due to Trichophyton tonsurans (variety sulfureum). Arch Dermatol 1951;63:493-499.

6. Mackenzie DWR, Burrows D, Walby AL. Trichophyton sulphureum in a residential school. Br Med J 1960;2:1055-1058.

7. Snowden MS, Loder L, Alexander WJ. Infectious alopecia in a child day-care center. JAMA 1985;254:3038.

8. Stiller MJ, Klein WP, Dorman RI, Rosenthal S. Tinea corporis gladitorium: an epidemic of Trichophyton tonsurans in student wrestlers. J Am Acad Dermatol 1992;27:632-633.

9. Bronson DM, Desai DR, Barsky S, Folley SM. An epidemic of infection with Trichophyton tonsurans revealed in a 20-year survey of fungal infections in Chicago. J Am Acad Dermatol
1983;8:322-330

10. Kane J, Leavitt E, Summerbell RC, Krajden S, Kasatiya SS. An outbreak of Trichophyton tonsurans dermatophytosis in a chronic care institution for the elderly. Eur J Epidemiol 1988;4:144-149.

11. Arnow PM, Houchins SG, Pugliese G. An outbreak of tinea corporis in hospital personnel caused by a patient with Trichophyton tonsurans infection. Pediatr Infect Dis J 1991;10:355-359.

12. Hakendorf AJ. An outbreak of Trichophyton rubrum infection in South Australia. Australas J Dermatol 1952;1:208-212.

13. Peachey RDG, English MP. An outbreak of Trichophyton rubrum infection in a geriatric hospital. $\mathrm{Br} J$ Dermatol 1974;91:389-397.

14. Shah PC, Kradjen S, Kane J, Summerbell RC. Tinea corporis caused by Microsporum canis: report of a nosocomial outbreak. Eur J Epidemiol 1988;4:33-38.

15. Rodriguez-Contreras PR, Delgado FV, Ayudarte MD, Cueto EA, Galvez VR. Nosocomial infections with Microsporum canis. J Hosp Infect 1987;9:201-202.

16. Garner JS, Simmons BP. CDC guideline for isolation precautions in hospitals. Infect Control 1983;4:245-325.

17. Garner JS, the Hospital Infection Control Practices Advisory Committee. Guideline for isolation precautions in hospitals.

\section{Hepatitis G: Not Quite Another Step in the Alphabet}

\section{Gina Pugliese, RN, MS Martin S. Favero, $\mathrm{PhD}$}

Approximately $10 \%$ to $15 \%$ of patients with parenterally transmitted non- $A$, non-B hepatitis have no evidence of hepatitis $\mathrm{C}$ virus (HCV) infection and are classified as having non-A, B, C, D, E, or non-A-to-E, hepatitis. Compared to patients with acute $\mathrm{HCV}$, patients with acute non-Ato-E hepatitis are less likely to be jaundiced, have lower peak alanine aminotransferase levels, and have a lower frequency of chronic hepatitis. Recently, a newly discovered virus isolated from patients with hepatitis, hepatitis $\mathrm{G}$ virus (HGV), has been described. Dr. Miriam J. Alter and coinvestigators from the CDC's Hepatitis Branch conducted a study to determine the characteristics of acute non-A-to-E hepatitis in the United
States and the possibility that it is caused by HGV. Using the sentinel surveillance system, the Hepatitis Branch has operated since 1982 , involving counties in four states. Patients were selected who had acute disease during 1985 to 1986 or 1991 to 1995. Serum samples were tested for HGV ribonucleic acid (RNA) by polymerase chain reaction. Hepatitis $G$ virus RNA was detected in 4 of 45 patients with a diagnosis of non-A-toE hepatitis (9\%), 23 patients with HCV (20\%), 25 of 100 patients with hepatitis A (25\%), and 32 of 100 patients with hepatitis B (32\%). The clinical characteristics of the acute illness were similar for patients with $\mathrm{HGV}$ alone and those with hepatitis $\mathrm{A}, \mathrm{B}$, or $\mathrm{C}$ with or without HGV infection. During a follow-up period of 1 to 9 years, chronic hepatitis did not develop in any of the patients with HGV alone, but 75\% were persistently positive for $\mathrm{HGV}$ RNA, as were $87 \%$ of those with both $\mathrm{HCV}$ and HGV infection. The rates of chronic hepatitis were similar in patients with $\mathrm{HCV}$ alone (60\%) and those with both HCV and HGV infection $(61 \%)$. It was concluded that HGV may not be a hepatotropic agent, but may induce hepatitis only under certain circumstances, as seen with other viruses such as cytomegalovirus and yellow fever virus. HGV was not implicated as an etiologic agent of non-Ato-E hepatitis. Persistent infection with HGV was common, but it did not lead to chronic disease and did not affect the clinical course or patients with hepatitis $\mathrm{A}, \mathrm{B}$, or $\mathrm{C}$.

FROM: Alter MJ, Gallagher M, Morris T, et al. Acute non-A-to-E hepatitis in the United States and the role of hepatitis G infection. $N$ Engl J Med 1997;336:741-746. 